\title{
Management challenges associated with a pineal region chordoma: illustrative case
}

\author{
Ji-Eyon Kwon, MD, ${ }^{1,3}$ So Young Ji, MD, ${ }^{1,3}$ Kihwan Hwang, MD, ${ }^{1,3}$ Kyu Sang Lee, MD, ${ }^{2}$ Gheeyoung Choe, MD, ${ }^{2}$ \\ Chae-Yong Kim, MD, ${ }^{1,3}$ and Jung Ho Han, MD ${ }^{1,3}$
}

\begin{abstract}
Departments of ${ }^{1}$ Neurosurgery and ${ }^{2}$ Pathology, Seoul National University Bundang Hospital, Gyeonggi-do, South Korea; and ${ }^{3}$ Department of Neurosurgery, Seoul National University College of Medicine, Seoul, South Korea
\end{abstract}

\begin{abstract}
BACKGROUND Chordomas, which are rare malignant neoplasms arising from notochordal remnants, often cause gradually progressive clinical symptoms. Intradural cranial chordomas (ICCs) are extremely rare and generally have a favorable prognosis. However, the authors reported the case of a primary ICC originating in the pineal gland presenting with recurrent thalamic hemorrhage and displaying an aggressive postoperative clinical course.

OBSERVATIONS A 41-year-old man arrived at the emergency department with morning headaches and recurrent syncope that had lasted several months. Computed tomography and magnetic resonance imaging (MRI) revealed a pineal gland mass causing obstructive hydrocephalus and a subacute hematoma in the right thalamus. Three weeks after an endoscopic third ventriculostomy was performed, recurrent hemorrhage was observed in the right thalamus. The tumor was promptly removed surgically. The yellowish-white tumor did not exhibit abundant bleeding. No evidence of intratumoral hemorrhage around the hematoma pocket was found. Histopathological examination revealed the characteristics of a chordoma with minimal vascularity. MRI performed 10 weeks postoperatively for worsening headaches revealed abnormal enhancement of multiple cranial nerves, suggesting leptomeningeal seeding (LMS) of the tumor.
\end{abstract}

LESSONS Despite radiotherapy and intrathecal chemotherapy, the patient's neurological status worsened; he died 2 years postoperatively. A pineal ICC may cause recurrent thalamic hemorrhage and potentially fatal LMS, even in the early postoperative period.

https://thejns.org/doi/abs/10.3171/CASE21110

KEYWORDS chordoma; pineal gland; thalamus; intracerebral hemorrhage; leptomeningeal seeding

Chordomas are rare malignant neoplasms that arise from notochordal remnants along the axial skeleton ${ }^{1}$ and usually present with gradually progressive clinical symptoms secondary to growth and bone destruction. ${ }^{2}$ Approximately $50 \%$ of all chordomas occur in the sacrococcygeal area, $30 \%$ occur in the skull base, and $20 \%$ occur in the mobile spine. ${ }^{3}$ Intradural cranial chordomas (ICCs) are extremely rare and mostly located in the prepontine and parasellar areas. ${ }^{4}$ On the other hand, the prognosis of ICCs is generally better than those of typical chordomas because their sharply circumscribed margins allow total excision. ${ }^{5,6}$ However, we report a rare case of a primary ICC originating in the pineal gland, presenting with recurrent thalamic hemorrhage and displaying an aggressive postoperative clinical course.

\section{Illustrative Case \\ Clinical Presentation}

A 41-year-old man presented at the emergency department with morning headaches and recurrent syncope that had lasted several months. A computed tomography (CT) scan revealed a mass-like lesion with eggshell-like high density of the pineal gland extending to the right thalamus as a low-density lesion and resulting in obstructive hydrocephalus (Fig. 1A). Magnetic resonance imaging (MRI) revealed a tumor of the pineal gland, which appeared iso/ hypointense on T1-weighted imaging (WI) and hyperintense on T2WI with heterogeneous contrast enhancement. The lesion in the right thalamus was identified as a subacute hematoma. A hypointense rim was also seen along the pineal tumor margin, especially

ABBREVIATIONS CT = computed tomography; ETV = endoscopic third ventriculostomy; ICC = intradural cranial chordoma; ICV = internal cerebral vein; LMS = leptomeningeal seeding; MRI = magnetic resonance imaging; PET = positron emission tomography; WI = weighted imaging.

INCLUDE WHEN CITING Published June 14, 2021; DOI: 10.3171/CASE21110.

SUBMITTED March 11, 2021. ACCEPTED April 26, 2021.

(C) 2021 The authors, CC BY-NC-ND 4.0 (http://creativecommons.org/licenses/by-nc-nd/4.0/). 

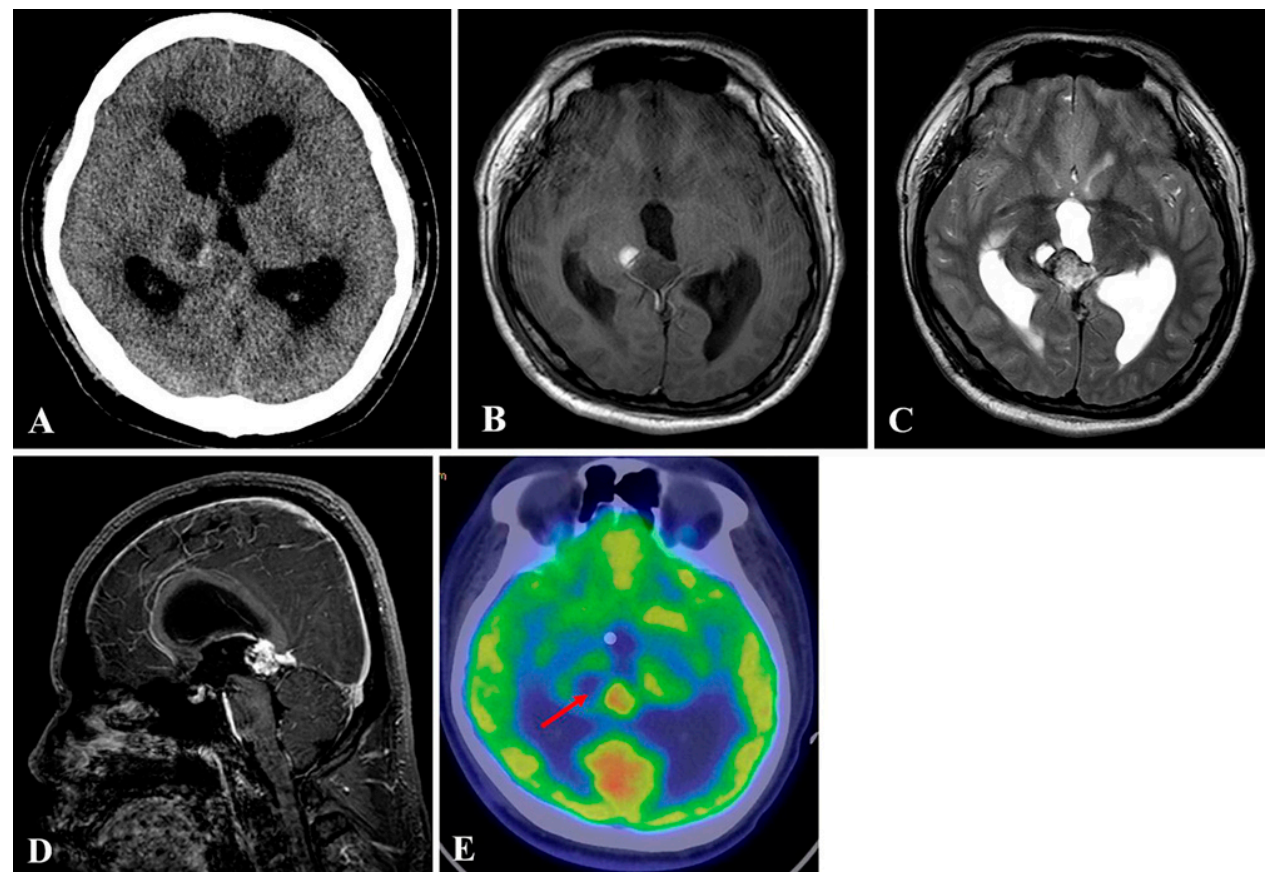

FIG. 1. CT scan reveals a mass-like lesion with eggshell-like high density of the pineal gland extending to the right thalamus as a low-density lesion (A). MRI shows a tumor of the pineal gland that appears iso/ hypointense on T1-WI (B) and hyperintense on T2-WI (C) with heterogeneous contrast enhancement (D). The lesion in the right thalamus was identified as a subacute hematoma. E: PET with 2-deoxy-2-[fluorine-18]fluoro-D-glucose shows a hypermetabolic lesion of the pineal gland (arrow); the right thalamic lesion was identified as a metabolic defect.

along the anterior and right sides on T2-WI (Fig. 1B-D). Positron emission tomography (PET) with 2-deoxy-2-[fluorine-18]fluoro-D-glucose revealed a hypermetabolic lesion of the pineal gland. On the other hand, the right thalamic lesion was identified as a metabolic defect (Fig. 1E). Cerebrospinal fluid testing did not reveal any abnormal findings. Based on these findings, the initial diagnosis was a pineal parenchymal tumor with a rare tumor bleed.

\section{Surgical Procedure}

Initially, endoscopic third ventriculostomy (ETV) was performed to resolve the obstructive hydrocephalus. Subsequently, resection of the tumor was planned, and the patient was discharged. Three weeks after the ETV, the patient presented at the emergency department again with a sudden headache. A CT scan revealed a newly developed hematoma at the site of the previous hematoma in the right thalamus (Fig. 2A). Prompt surgery to remove the tumor was planned because the tumor seemed to be the cause of the recurrent bleeding. MRI was performed to aid in intraoperative navigation. Serial T2-WI revealed narrowing of the right internal cerebral vein (ICV) and an acute phase hematoma in the right thalamus (Fig. 2B and C).

The surgery was performed via an occipital transtentorial approach. The tumor was yellowish white, and bleeding from the tumor was not abundant. However, it was hard and tough and densely adhered to both ICVs, especially on the right side. Thus, it could not be easily removed either with a tumor forceps or with an ultrasonic aspirator. On the right side of the tumor, the hematoma pocket in the right thalamus was identified and evacuated. However, there was no evidence of bleeding in the tumor itself around the hematoma pocket. A subtotal resection was performed, leaving the anterosuperior part of the tumor intact to save the bilateral ICVs (Fig. 3A).

\section{Histopathological Findings}

The gross histopathology revealed chords, sheets, and individual cells with bubbly cytoplasm (physaliphorous cells) arranged in
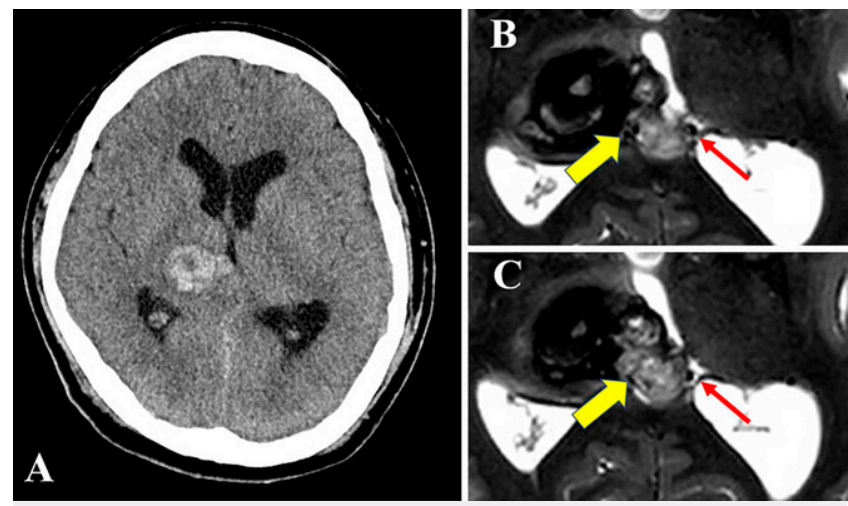

FIG. 2. A: CT scan shows a newly developed hematoma at the site of the previous hematoma in the right thalamus. B and C: MRI was performed for intraoperative navigation; serial T2-WI shows narrowing of the lumen of the right ICV (yellow arrows) compared to that of the left ICV (red arrows) and an acute phase hematoma in the right thalamus. 

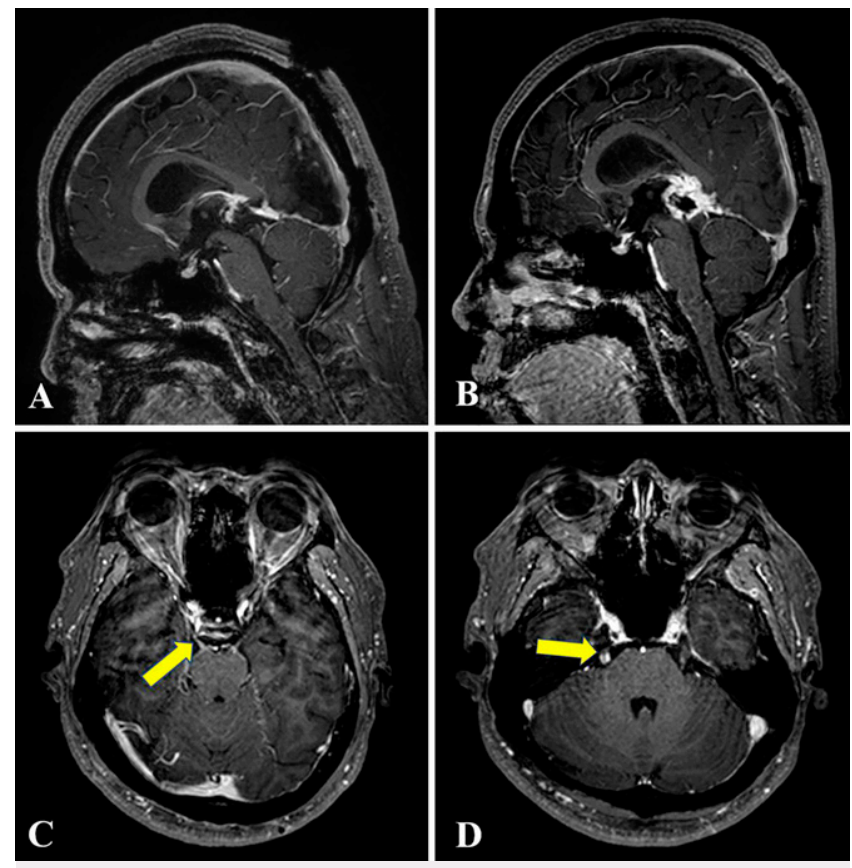

FIG. 3. A subtotal resection was performed, leaving the anterosuperior part of the tumor intact to save the bilateral ICVs (A). Follow-up MRI performed 10 weeks after the surgery reveals interval growth of the residual tumor $(\mathbf{B})$ and abnormal enhancement of the cranial nerves, including the bilateral third nerves (arrow in $\mathbf{C}$ ) and trigeminal nerves (arrow in D), suggesting LMS of the tumor (C and D).

lobules set within a myxochondroid background. Increased cellularity and moderate nuclear polymorphism were also seen. Mitotic activity and necrosis were not definitively identified. The diagnosis of chordoma or chondrosarcoma was initially considered. Immunohistochemistry revealed that the tumor cells were reactive for both $\mathrm{S} 100$ and cytokeratin (Fig. 4). The final histopathological diagnosis was a chordoma with minimal vascularity and no definite evidence of intratumoral hemorrhage.

\section{Postoperative Course}

Proton beam radiotherapy was planned for treatment of the residual tumor, and the patient was discharged in the absence of any definite neurological abnormalities. Thereafter, the patient repeatedly visited the outpatient clinic or the emergency department for a progressively worsening headache 6 weeks after the tumor resection. A follow-up MRI performed 10 weeks after the surgery revealed interval growth of the residual tumor (Fig. 3B) and abnormal enhancement of the cranial nerves, including the bilateral third nerves, trigeminal nerves, and facial nerves, suggesting leptomeningeal seeding (LMS) of the tumor (Fig. 3C and D).

Whole-brain radiotherapy with a dose of 3,000 cGy was applied in 10 fractionations with an additional boost of 2,000 cGy of radiotherapy to the primary site. Next, the administration of intrathecal methotrexate was commenced. After two trials of intrathecal methotrexate, whole-spine radiotherapy with a total dose of 4,000 cGy was applied in 20 fractionations for the thoracolumbar area. A total of 31 trials of intrathecal methotrexate was performed in 2 years, and these procedures induced necrotizing leukoencephalopathy.

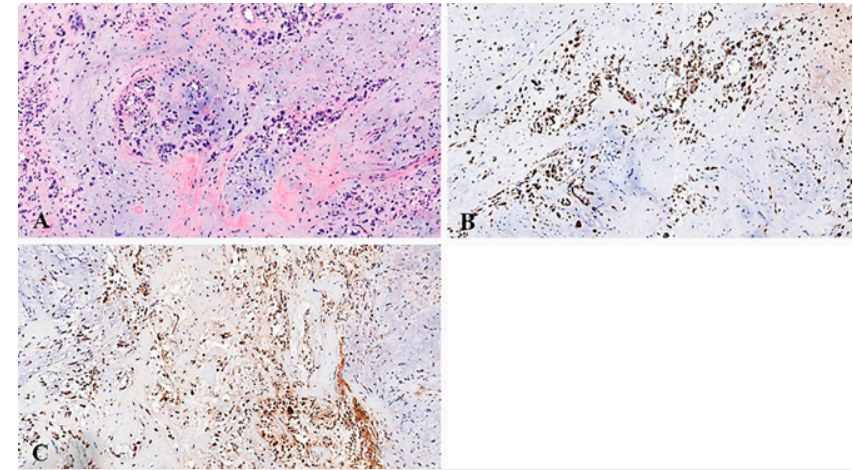

FIG. 4. Hematoxylin and eosin staining (original magnification $\times 200$ ) shows chords, sheets, and individual cells with bubbly cytoplasm (physaliphorous cells) arranged in lobules set within a myxochondroid background (A). Immunohistochemistry reveals that the tumor cells are reactive for both $\mathrm{S} 100(\mathrm{~B})$ and cytokeratin $(\mathrm{C})$. The final histopathological diagnosis is a chordoma.

Despite the abovementioned treatments, including ventriculoperitoneal shunting for controlling intracranial pressure, the patient's neurological status worsened, and he died 2 years after the tumor resection.

\section{Discussion}

\section{Observations}

Pineal Chordoma and Recurrent Bleeding

The key finding in this case was that a pineal ICC may cause recurrent thalamic hemorrhage and potentially fatal LMS, even in the early postoperative period. ICCs are rare, and to date, only approximately 60 cases have been reported. ${ }^{4}$ Moreover, ICCs of the pineal gland are extremely rare. Only one case of a chordoma of the pineal gland confirmed by autopsy has been reported, which was initially diagnosed as a germinoma. ${ }^{7}$ Before initiation of involution at approximately the 6th or 7th week of embryonic life, the notochord extends from the most caudal end of the vertebral column and terminates in what will develop into the dorsum sellae of the skull base. ${ }^{1}$ Thus, the finding of a chordoma of the pineal gland is unexpected. Rests of notochordal cells have been found in the prepontine extradural space posterior to the clivus, ${ }^{8}$ which is consistent with the spatial distribution of chordomas. ${ }^{1}$ Further research is necessary to identify how far these notochordal vestiges can be found in the brain.

The symptoms of intracranial chordoma vary with the location of the lesion and its proximity to critical structures ${ }^{9}$ and gradually progress because of tumor growth and bone destruction. However, although rare, cases of bleeding from a chordoma abruptly exacerbating neurological status have been reported. ${ }^{10-12}$ The cause of tumor hemorrhage in such cases remains to be elucidated. Intratumoral hemorrhage in chordomas may result from the rupture of thin-walled vessels, hemorrhagic necrosis due to rapid tumor growth, or destruction of the dural vessels by tumor invasion. ${ }^{10,12}$ However, we could not identify any evidence of tumor bleeding during surgery in this case, and there was no histopathological evidence of intratumoral bleeding. Failure of the venous drainage of the right thalamus could reasonably be assumed to have been the 
cause of recurrent bleeding in this case considering the narrowing or collapse of the right ICV by the firm and hard tumor.

\section{Lessons}

LMS of ICCS

In previous case reports and small case series, ICCs were generally considered to have a more favorable prognosis and a different biological behavior relative to those of typical skull base chordomas. ${ }^{2,13}$ However, recent data suggest that the overall prognosis of ICCs may be comparable to those of typical chordomas, although gross-total resection may be more feasible for true intradural chordomas. ${ }^{2}$ Moreover, ICCs present an additional problem: chordomas with intradural extension are at risk of LMS along the subarachnoid space. ${ }^{1}$ In fact, there have been reports of LMS or drop metastases of chordomas after surgery, as in the case reported in this paper. ${ }^{4,14-21}$ The proximity of the lesions to the cerebrospinal fluid space and/or ventricular system and intraventricular exposure during surgery may increase the risk of LMS. ${ }^{4}$ Although LMS usually develops as an end-stage event several years after surgery, ${ }^{15,20}$ it can occur in the early postoperative period, even within a week of surgery. ${ }^{14}$ In the present case, the headache that had ceased after surgery recurred within 6 weeks of the tumor resection. The follow-up MRI performed 10 weeks after surgery to identify the cause of the unresolved headaches confirmed LMS. In situations in which there is no clear evidence of whether radiotherapy will be effective in preventing LMS, as in this case, it is difficult to be sure if LMS could have been prevented had the adjuvant treatment commenced earlier. Therefore, it is important to bear in mind that LMS can occur even in the early postoperative period, and careful and timely follow-ups and optimal scheduling of the adjuvant treatment are necessary, especially in patients who exhibit postoperative neurological symptoms or unresolved headaches.

\section{Conclusions}

ICCs are rare tumors that generally have a favorable prognosis. However, recurrent thalamic bleeding from a pineal ICC may occur as the result of the failure of venous drainage, and potentially fatal LMS can occur even in the early postoperative period. Therefore, careful and timely follow-ups and optimal scheduling of the adjuvant treatment are necessary, especially in patients who exhibit postoperative neurological symptoms or unresolved headaches.

\section{References}

1. George B, Bresson D, Herman P, Froelich S. Chordomas: a review. Neurosurg Clin N Am. 2015;26(3):437-452.

2. Al Otaibi F, Guiot MC, Muanza T, Di Maio S. Giant petroclival primary intradural chordoma: case report and systematic review of the literature. J Neurol Surg Rep. 2014;75(1):e160-e169.

3. Zeng HH, Yu XB, Fan LF, Chen G. Diagnostic clues and treatment of intradural cranial chordoma. Chin Med J (Engl). 2016;129(23): 2895-2896.

4. Rinaldo L, Priemer DS, Vortmeyer AO, et al. Chordoma of the corpus callosum: case report. J Neurosurg. 2018;131:1380-1386.

5. Choo YS, Joo SW, Noh SJ, Lee SI. Intradural retroclival chordoma. J Korean Neurosurg Soc. 2009;46(2):152-155.

6. Masui K, Kawai S, Yonezawa T, et al. Intradural retroclival chordoma without bone involvement: case report. Neurol Med Chir (Tokyo). 2006;46(11):552-555.
7. Figueiredo EG, Tavares WM, Welling $L$, et al. Ectopic pineal chordoma. Surg Neurol Int. 2011;2:145.

8. Mehnert F, Beschorner R, Küker W, et al. Retroclival ecchordosis physaliphora: MR imaging and review of the literature. AJNR Am J Neuroradiol. 2004;25(10):1851-1855.

9. Erdem E, Angtuaco EC, Van Hemert R, et al. Comprehensive review of intracranial chordoma. Radiographics. 2003;23(4): 995-1009.

10. Kim SK, Kim YH, Park CK, et al. Intracranial intradural chordoma presenting with intraventricular hemorrhage. Clin Neurol Neurosurg. 2012;114(8):1189-1192.

11. Vellutini $E$, de Oliveira MF. Intradural chordoma presenting with intratumoral bleeding. J Clin Neurosci. 2016;25:139-142.

12. Mohindra S, Kapoor A, Kursa GK, et al. Apoplexy in an intradural clival chordoma causing intraventricular bleed. Surg Neurol Int. 2016;7(suppl 1):S17-S19.

13. Fracasso T, Brinkmann B, Paulus W. Sudden death due to subarachnoid bleeding from ecchordosis physaliphora. Int $J$ Legal Med. 2008;122(3):225-227.

14. Zhang J, Gao CP, Liu XJ, Xu WJ. Intradural cervical chordoma with diffuse spinal leptomeningeal spread: case report and review of the literature. Eur Spine J. 2018;27(suppl 3):440-445.

15. Vellutini EAS, Brock RS, Martins HO, et al. Diffuse spinal spreading following previous intracranial intradural chordoma resection: a rare case report. J Clin Neurosci. 2019;64:44-46.

16. Stough DR, Hartzog JT, Fisher RG. Unusual intradural spinal metastasis of a cranial chordoma. Case report. J Neurosurg. 1971;34(4):560-562.

17. Martin MP, Olson S. Intradural drop metastasis of a clival chordoma. J Clin Neurosci. 2009;16(8):1105-1107.

18. Korinth M, Schönrock L, Mayfrank L, Gilsbach JM. Primary intradural pontocerebellar chordoma metastasizing in the subarachnoid spinal canal. Zentralbl Neurochir. 1999;60(3): 146-150.

19. Ji T, Guo W, Shen DH, et al. Intradural spinal seeding and fatal progression of a sacrococcygeal chordoma: a case report. Chin Med J (Engl). 2008;121(12):1150-1152.

20. Champeaux K, San-Galli F, Eimer S, Liguoro D. Chordoma radicular metastasis following cerebrospinal fluid dissemination. Article in French. Neurochirurgie. 2008;54(1):41-45.

21. Asano $S$, Kawahara N, Kirino T. Intradural spinal seeding of a clival chordoma. Acta Neurochir (Wien). 2003;145(7):599-603.

\section{Disclosures}

The authors report no conflict of interest concerning the materials or methods used in this study or the findings specified in this paper.

\section{Author Contributions}

Conception and design: Han, Kwon, Kim. Acquisition of data: Han, Kwon, Ji, Hwang, Lee, Choe. Analysis and interpretation of data: Han, Kwon, Choe. Drafting the article: Han, Kwon. Critically revising the article: Han, Kwon, Choe. Reviewed submitted version of manuscript: Han, Kwon, Ji, Choe, Kim. Approved the final version of the manuscript on behalf of all authors: Han. Administrative/technical/material support: Ji, Hwang, Choe. Study supervision: Ji, Kim.

\section{Correspondence}

Jung Ho Han: Seoul National University Bundang Hospital, Gyeonggido, Republic of Korea. nstaus29@snu.ac.kr. 